DESY $99-130$

HLRZ 99-39

September 1999

\title{
Lattice chiral fermions in the background of non-trivial topology*
}

\author{
V. Bornyakov ${ }^{\mathrm{a}}$, A. Hoferichter ${ }^{\mathrm{b}}$, G. Schierholz ${ }^{\mathrm{b}, \mathrm{c}}$, and A. Thimm ${ }^{\mathrm{b}, \mathrm{d}}$ \\ anstitute for High Energy Physics IHEP, RU-142284 Protvino, Russia \\ ${ }^{\mathrm{b}}$ Deutsches Elektronen-Synchrotron DESY and NIC, D-15735 Zeuthen, Germany \\ ${ }^{c}$ Deutsches Elektronen-Synchrotron DESY, D-22603 Hamburg, Germany \\ ${ }^{\mathrm{d} I n s t i t u t ~ f u ̈ r}$ Theoretische Physik, Freie Universität Berlin, D-14195 Berlin, Germany
}

We address the problem of numerical simulations in the background non-trivial topology in the chiral Schwinger model. An effective fermionic action is derived which is in accord with established analytical results, and which satisfies the anomaly equation. We describe a numerical evaluation of baryon number violating amplitudes, specifically the 't Hooft vertex.

\section{CFA to chiral gauge theories}

The main idea of the Continuum Fermion Approach (CFA) [1] to chiral gauge theories is to discretize the gauge fields only, and to consider the fermions in the continuum. In order to do so, we need to extrapolate the lattice gauge fields to continuum gauge fields [5].

The generating functional for $\mathrm{LH}$ fermions in the sector of non-trivial topology with charge $Q \leq$ 0 is defined by

$$
\begin{aligned}
& Z(\eta, \bar{\eta})=\int \mathcal{D} U \exp \left(-S_{G}\right) Z(A, \eta, \bar{\eta}), \\
& Z(A, \eta, \bar{\eta})=\int \mathcal{D} \psi \mathcal{D} \bar{\psi} \exp \left(-S_{F}+\bar{\psi} \eta+\bar{\eta} \psi\right) \\
& \equiv \exp (-W(A)) \exp (\bar{\eta} G \eta) \\
& \times \prod_{i=1}^{|Q|}<\bar{\eta} \phi_{i}>,
\end{aligned}
$$

where $S_{G}(U)$ and $S_{F}(A, \bar{\psi}, \psi)$ are the lattice gauge field action and the continuum fermion action, respectively, with $U=\exp \left(\mathrm{i} e \int A\right)$. The $\phi_{i}$ 's are the zero mode eigenfunctions, and $G$ is the Green function orthogonal to the eigenfunctions' subspace. Whenever we write $A$ we mean the lattice gauge field extrapolated to the continuum.

The $U$ 's live on a lattice with lattice spacing

\footnotetext{
*Talk presented by V. Bornyakov at Lattice '99, Pisa, Italy
}

a. In practice the fermions are put on a fine lattice with spacing $a_{f}$, with the understanding of finally taking $a_{f} \rightarrow 0$. Gauge invariance breaking effects can be made arbitrarily small by making $a_{f} / a$ small enough so that they do not affect the continuum limit $a \rightarrow 0$ (in accordance with [6]). The effective action $W(A)$ is defined as $[7]$

$$
\begin{aligned}
W(A)= & \lim _{a_{f} \rightarrow 0} W\left(U^{f}\right), \\
W\left(U^{f}\right)= & -\log \operatorname{det} D\left(U^{f}\right) \\
& + \text { local counterterms, }
\end{aligned}
$$

where $U^{f}$ is the link variable on the fine lattice, with (for one species of LH fermions)

$D\left(U^{f}\right)=\not D\left(U^{f}\right) P_{L}+\not D(1) P_{R}+W$,

$W$ being the Wilson term. For background fields with trivial topology it has been demonstrated, both analytically and numerically, that $W(A)$ exists having all desired properties. In particular,

$\operatorname{Re} W(A)=\frac{1}{2}\left(W_{V}(A)+W_{0}\right)$,

where $W_{V}\left(W_{0}\right)$ is the effective action of the vector (free) theory. Furthermore, $W(A)$ gives the right anomaly, and $\operatorname{Im} W(A)$ is gauge invariant in the anomaly free model. We also did not encounter any problems with large (singular) gauge transformations 8, which cause trouble in the overlap approach 10] (and presumably in Slavnov's approach [11] as well). 
Using Neuberger's operator [12] instead of the Dirac-Wilson operator, Re $W$ can be computed on the original lattice due to the absence of additive fermion mass renormalization.

\section{Evaluation of 't Hooft vertex}

The formal continuum expression for the 't Hooft vertex in the anomaly free CSM with four LH fermions of charge 1 and one $\mathrm{RH}$ fermion of charge 2 is

$$
<H(x)>=\frac{\int \mathcal{D} A \mathcal{D} \psi \mathcal{D} \bar{\psi} \exp \left(-S_{G}-S_{F}\right) H(x)}{\int \mathcal{D} A \mathcal{D} \psi \mathcal{D} \bar{\psi} \exp \left(-S_{G}-S_{F}\right)},
$$

where

$\mathcal{D} \psi=\left(\prod_{i=1}^{4} \mathcal{D} \psi_{i}^{(1)}\right) \mathcal{D} \psi^{(2)}$,

and similarly for $\mathcal{D} \bar{\psi}$, and where

$H(x)=\frac{\pi^{2}}{e^{4}}\left(\prod_{i=1}^{4} \psi_{i, L}^{(1)}(x)\right) \bar{\psi}_{R}^{(2)}(x) \not \partial \bar{\psi}_{R}^{(2)}(x)$.

The integral in the numerator is over the sector with topological charge $Q=-1$, while that in the denominator is over the sector with $Q=0$.

On the $l \times l$ torus the gauge field takes the form

$A_{\mu}(x)=\frac{2 \pi}{l} t_{\mu}+\partial_{\mu} h(x)+\varepsilon_{\mu \nu} \partial_{\nu} \alpha(x)+C_{\mu}^{Q}(x),(10)$

where $t_{\mu}$ is the toron field, $\partial_{\mu} h$ represents the gauge degrees of freedom, and $\varepsilon_{\mu \nu} \partial_{\nu} \alpha$ and $C_{\mu}^{Q}$ are the proper dynamical fields of zero and nonzero topological charge, respectively.

After integration over the fermion fields and change of variables, eq. (7) becomes

$$
<H(x)>=\frac{\int \mathcal{D} \alpha \mathcal{D} t \exp \left(-S_{G}^{N}-W_{N}\right) H^{0}(x)}{\int \mathcal{D} \alpha \mathcal{D} t \exp \left(-S_{G}-W_{D}\right)}
$$

where 13, 14,9, 15

$$
\begin{array}{r}
H^{0}(x)=\frac{\pi^{2}}{e^{4}}\left(\prod_{i=1}^{4} \phi_{i}^{(1)}(x)\right)\left(\phi_{1}^{(2) \dagger}(x) \sigma_{\mu} \partial_{\mu} \phi_{2}^{(2) \dagger}(x)\right. \\
\left.-\phi_{2}^{(2) \dagger}(x) \sigma_{\mu} \partial_{\mu} \phi_{1}^{(2) \dagger}(x)\right) .
\end{array}
$$

In eq. (11)

$S_{G}^{N}=S_{G}+\frac{2 \pi^{2}}{e^{2} l^{2}}, S_{G}=\frac{-1}{2 e^{2}} \int \mathrm{d} y \alpha(y) \square^{2} \alpha(y),($ and

$$
\begin{aligned}
& W_{N, D}(A)=4 W_{N, D}^{(1)}(A)+W_{N, D}^{(2)}(A), \\
& W_{D}^{(k)}(A)=W^{(k)}(\alpha)+W^{(k)}(t), \\
& W_{N}^{(k)}(A)=W^{(k)}(\alpha)+W_{\phi}^{(k)} \\
& (k=1,2), \text { with } \\
& W^{(k)}(\alpha)=-\frac{k^{2}}{4 \pi} \int \mathrm{d} y \alpha(y) \square \alpha(y), \\
& W_{\phi}^{(k)}=-\log \left(\left(\frac{2 k}{l^{2}}\right)^{k / 2} \operatorname{det}\left(<\phi^{(k)}, \phi^{(k)}>\right)\right) .
\end{aligned}
$$

The 't Hooft vertex $<H(x)>$ has been computed analytically in 13. with the help of results obtained for the Schwinger model in 14.

In this paper we present results obtained in a 'hybrid' calculation. For the fermionic expressions we use the analytically known formulae, which is equivalent to taking the limit $a_{f}=0$ in the CFA. In this way we by-pass the costly calculation of the fermionic determinant on ever larger lattices. The integration over the gauge fields is done numerically. It has been shown that the $t$-dependence factorizes out in both, the numerator and the denominator, and that the factors cancel each other if one adopts special fermionic boundary conditions 13 . We then find for the numerator

$$
\begin{gathered}
N=\frac{C}{Z(\alpha)} \int \prod_{n} \mathrm{~d} \alpha(n) \exp \left(\frac{-\beta}{2} \sum_{n}(\square \alpha(n))^{2}\right) \\
\times \exp (-W(\alpha)-8 \alpha(x)),
\end{gathered}
$$

and for the denominator

$$
\begin{gathered}
D=\frac{1}{Z(\alpha)} \int \prod_{n} \mathrm{~d} \alpha(n) \exp \left(\frac{-\beta}{2} \sum_{n}(\square \alpha(n))^{2}\right) \\
\times \exp (-W(\alpha)),
\end{gathered}
$$

where

$$
Z(\alpha)=\int \prod_{n} \mathrm{~d} \alpha(n) \exp \left(\frac{-\beta}{2} \sum_{n}(\square \alpha(n))^{2}\right)
$$

and

$C=\frac{64 \pi}{(m l)^{4}} \exp \left(-\frac{8 \pi}{(m l)^{2}}\right) \eta^{8}(1)$.

Here $m$ is the gauge boson mass, with $m^{2}=$ $4 e^{2} / \pi=4 / a^{2} \pi \beta$, and $\eta$ is Dedekind's function. 
The simulations were done for $m l=3$ and 4 on lattices of size $L=l / a$ varying from 12 to 48 . In Fig. 1 we show our results as a function of $1 / L^{2}$. Also shown is the analytic result [13]. We find excellent agreement between our results extrapolated to $L=\infty$ and the analytic values.

\section{Conclusions}

We have once more illustrated that the CFA is a powerful tool for formulating and analyzing chiral gauge theories on the lattice. In particular, we find stable results at large values of $L$, which allow us to determine the continuum numbers rather accurately. A problem of the overlap approach is 13] that a Thirring term is generated dynamically, which has to be tuned carefully so that its effective coupling vanishes. This is particularly aggravating at large $L$, and so far has prevented a reliable extrapolation to the continuum. In our approach, being intrinsically gauge invariant, this problem does not exist. A totally numerical computation of the 't Hooft vertex is under way.

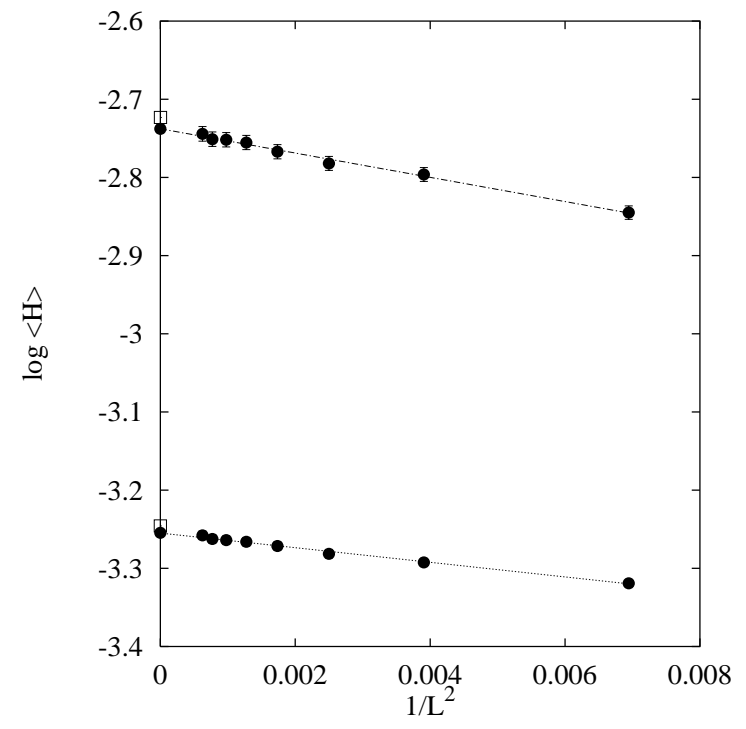

Figure 1. The 't Hooft vertex for two parameter sets, $m l=4$ (top) and 3 (bottom), together with the extrapolation to the continuum. This is compared to the analytic values $(\square)$.

\section{References}

1. L. Alvarez-Gaumé and S. Della-Pietra, in Recent Developments in Quantum Field Theory, eds. J.Ambjorn, B. J. Durhuus and J. L. Petersen (North-Holland, 1985).

2. M. Göckeler and G. Schierholz, Nucl. Phys. B (Proc. Suppl.) 29B,C (1992) 114, ibid. 30 (1992) 609.

3. G. 't Hooft, Phys. Lett. B349 (1995) 491.

4. P. Hernandez and R. Sundrum, Nucl. Phys. B455 (1995) 287; G. T. Bodwin, Phys. Rev. D54 (1996) 6497.

5. M. Göckeler, A. S. Kronfeld, G. Schierholz and U.-J. Wiese, Nucl. Phys. B404 (1993) 839.

6. D. Foerster, H. Nielsen and M. Ninomiya, Phys. Lett. B94 (1980) 135.

7. V. Bornyakov, G. Schierholz and A. Thimm, Nucl. Phys. B (Proc. Suppl.) 63 (1998) 593.

8. V. Bornyakov, G. Schierholz and A. Thimm, in Proceedings of 31st Intern. Symposium Ahrenshoop, Buckow, 1997, p. 265 (WileyVCH, Berlin, 1998) (hep-lat/9805007).

9. V. Bornyakov, G. Schierholz and A. Thimm, Prog. Theor. Phys. Suppl. 131 (1998) 337 (hep-lat/9806010).

10. R. Narayanan and H. Neuberger, Nucl. Phys. B477 (1996) 521; T. Aoyama and Y. Kikukawa, Nucl. Phys. B (Proc. Suppl.) 53 (1997) 638.

11. A. A. Slavnov and N. V. Zverev, Phys. Lett. B420 (1998) 323.

12. H. Neuberger, Phys. Lett. B417 (1998) 141; ibid. B427 (1998) 125.

13. Y. Kikukawa, R. Narayanan, H. Neuberger, Phys. Lett. B399 (1997) 105; Phys. Rev. D57 (1998) 1233.

14. I. Sachs and A. Wipf, Helv. Phys. Acta 65 (1992) 652.

15. L. Alvarez-Gaumé, G. Moore and C. Vafa, Commun. Math. Phys. 106 (1986) 1; M. Göckeler and G. Schierholz, unpublished (1994); R. Narayanan and H. Neuberger, Phys. Lett. B348 (1995) 549. 